\title{
Postsuburbia à Madrid. Fragmentation, réseaux et nœuds dans la périphérie rurbaine
}

Post-Suburbia in Madrid. Fragmentations, networks and nodes in the urbanrural periphery

Post-Suburbia in Madrid. Fragmentierung, Netze und Knoten in der urbanruralen Peripherie

\section{Rainer Wehrhahn}

\section{(Q) OpenEdition}

\section{Journals}

Édition électronique

URL : http://journals.openedition.org/rge/316

DOI : $10.4000 /$ rge. 316

ISSN : 2108-6478

\section{Éditeur}

Association des géographes de l'Est

Édition imprimée

Date de publication : 1 juin 2005

Pagination : 175-186

ISSN : 0035-3213

Référence électronique

Rainer Wehrhahn, « Postsuburbia à Madrid. Fragmentation, réseaux et nœuds dans la périphérie rurbaine ", Revue Géographique de l'Est [En ligne], vol. 45 / 3-4 | 2005, mis en ligne le 04 juin 2009, consulté le 08 septembre 2020. URL : http://journals.openedition.org/rge/316 ; DOI : https://doi.org/ $10.4000 /$ rge.316

Ce document a été généré automatiquement le 8 septembre 2020.

Tous droits réservés 


\section{Postsuburbia à Madrid.} Fragmentation, réseaux et nœuds dans la périphérie rurbaine

Post-Suburbia in Madrid. Fragmentations, networks and nodes in the urbanrural periphery

Post-Suburbia in Madrid. Fragmentierung, Netze und Knoten in der urbanruralen Peripherie

Rainer Wehrhahn

\section{NOTE DE L'ÉDITEUR}

Article reçu le 6 novembre 2005, accepté le 31 janvier 2006

Adaptation : Michel Deshaies

\section{Postsuburbia - concepts théoriques et état de la question}

1 Les périphéries des villes se sont profondément transformées au cours des dernières décennies. La phase de suburbanisation s'accentue encore par une dichotomie entre le noyau urbain et la périphérie. Ainsi découvrons nous aujourd'hui dans la totalité de l'agglomération, de tout nouveaux entrelacements et des liens bien plus complexes entre chacun des éléments de l'espace influencé par la ville. En raison de l'expansion d'îlots urbains dans des zones éloignées de l'environnement rural des agglomérations, il est important d'intégrer cette zone urbaine et rurale de contact dans les débats scientifiques et cela plus distinctement que ce qui a pu être fait jusqu'à présent. Continuum ville-campagne, "Zwischenstadt», post-suburbia - les désignations employées pour définir ces «nouveaux» espaces sont nombreuses, bien qu'aucun 
concept théorique ne soit communément accepté pour la description et l'explication du « processus d'urbanisation » dans cette zone.

Les sociologues nord-américains ont d'abord fait avancer la discussion conceptuelle autour des processus en cours dans les périphéries rurbaines : Michael Dear (2000) ou Dear et Flusty (1998) dans leurs œuvres de référence, ou encore Ed Soja dans sa monographie de 2000. Mais aussi Davis (1990) ou le journaliste Garreau (1991) comptent parmi les pionniers du développement de la théorie conceptuelle de la description et de l'explication de l'urbanisation post-moderne. Dear et Flusty (1998) ont présenté une vision de la structure des villes "post-modernes", qui a été reprise par d'autres auteurs (ex. Kunzmann 2001). Le processus principal est la « dissolution » des fonctions du noyau urbain en faveur d'une répartition de ces fonctions sur la totalité de l'espace des villes post-modernes. Il se produit donc une décentralisation, avec un étalement excessif de la surface urbaine/post-urbaine et simultanément une recentralisation avec la formation de nouveaux nœuds ou edge cities dans la post-suburbia.

Une transposition de ces idées dans d'autres contextes spatiaux et aussi dans les manuels de géographie urbaine a par exemple été effectuée par Hall (1998). Même si ce que l'on appelle l'école de Los Angeles avec ses diagnostics n'est en aucun cas incontestée et cela même aux Etats-Unis, certains éléments isolés sont également repris dans des contextes européens. Dans l'espace germanophone, ces interprétations ont été effectuées par des géographes comme par exemple Aring (1999), Wehrhahn $(2000,2001)$ ou Wood (2003). A peu près en même temps, d'autres scientifiques des domaines de l'architecture ou de la planification se sont consacrés à la question de savoir comment on peut interpréter les nouveaux processus dans les périphéries urbaines et quels défis ils représentent pour la pratique de la planification. Sieverts a inventé la notion de «Zwischenstadt» (Sieverts 1998), mais d'autres auteurs se sont également exprimés sur le sujet dans de nombreux recueils. Après l'achèvement de projets interdisciplinaires internationaux sur ce thème (voir Borsdorf et Zembri 2004, DuboisTaine 2004, Franzén et Halleux 2004), on dispose aujourd'hui de suffisamment d'indications pour l'adaptation de la structure des "outskirts» dans des contextes européens.

4 Sur cet arrière-plan, les évolutions et débuts d'explications suivantes serviront de point de départ conceptuel pour la classification théorique des recherches effectuées à Madrid :

1. À l'époque de la mondialisation, ce sont souvent les restructurations économiques qui déterminent les conditions des changements sur le plan national et régional. Pour ce qui est du temps et de l'espace, ce sont les procédés de production postfordistes et les conditions de la distribution qui définissent les marges d'action des acteurs économiques urbains. La contrainte de la flexibilité se traduit dans les structures spatiales dans la mesure où les décisions d'investissements économiques constituent des points d'ancrage plus ou moins durables qui façonnent la périphérie. Viennent s'y ajouter de nouvelles formes de consommation d'aventure et d'organisation des loisirs qui influencent également, dans des proportions considérables, le développement de la structure des espaces périphériques.

2. Le changement social se caractérise par une modification des classes et des couches sociales où l'individualisme et l'hétérogénéité sociale sont plus importants. Cela a pour conséquence une fragmentation spatiale qui se manifeste dans la périphérie par d'extrêmes différences dans les quartiers d'habitation et dans les façons d'y habiter. En revanche, les gated communities qui se constituent suivant des formes très variées n'en sont qu'une expression possible. Constituant l'une des motivations les plus déterminantes de la fragmentation des 
structures d'habitation, elles donnent lieu à la réalisation de "nouveaux " modes de vie, contrairement à la suburbanisation classique qui elle, se caractérise par une structure uniforme.

3. Le passage de la suburbanisation à la post-suburbia a provoqué des changements démographiques et socio-culturels : il n'y a plus d'homogénéité en ce qui concerne les structures d'âge ou de revenus, mais plutôt un modèle démographique hétérogène qui se différencie encore plus par les migrations nationales et internationales. L'hétérogénéité culturelle n'est plus seulement propre aux centres urbains mais elle s'étend aussi aux périphéries.

4. Finalement, ce sont de nouvelles formes de politique culturelle et de planification qui s'imposent, incluant beaucoup plus de composantes économiques privées et que l'on peut résumer comme étant la substitution d'une urban governance à l'urban government autrefois plus actif.

5 L'analyse de la post-suburbia est de plus compliquée par le fait que tous les processus mentionnés se contrebalancent. En outre, dans ce contexte compliqué, la question se pose de savoir comment peser la part relative des facteurs de différentes échelles et s'ils s'influencent mutuellement. Il est évident que cette étude n'apporte pas de réponse complète à la problématique. C'est pourquoi, à l'aide d'exemples, on essaiera d'apporter simplement un éclairage aux questions complexes suivantes :

- Existe-t-il d'une manière générale à Madrid, de nouvelles structures dans la périphérie urbaine (ou mieux encore : la périphérie urbano-rurale) ? Quelque chose devant être désigné avec le préfixe « post » vient-il après la suburbanisation? A quoi ressemblent concrètement ces structures, sont-elles effectivement établies sous forme d'îlots et quelles fonctions remplissent-elles? Autrement dit : Est-il réellement question ici de fragments, les trouve-ton dispersés ou est-ce que l'on doit définir la posturbia plutôt comme une « solution » (salad bowl ou melting pot)?

- La postsuburbia constitue-t-elle une « ville réseau » et dans ce cas, peut-on y trouver des nœuds qui seraient en contact les uns avec les autres par des interconnexions? Les structures résidentielles madrilènes sont-elles vraiment à la fois dispersées et polycentriques comme c'est le cas dans les périphéries post-modernes?

- Quelles sont les approches pertinentes pour expliquer l'évolution des périphéries au niveau des macrostructures (est-ce qu'alors ce sont les structures et les processus économiques, socio-culturels et politiques qui sont au premier plan? Et quels actes individuels, par exemple de personnes y transférant leur résidence principale ou les acteurs centraux de l'aménagement des villes, y déterminent-ils la restructuration de la périphérie?

Dans une première partie de l'analyse empirique, on présente sur la base des statistiques des unités spatiales de la région métropolitaine, les résultats concernant l'hétérogénéisation et la fragmentation des rapports ; on prend ainsi en compte non seulement les données socioéconomiques et démographiques, mais aussi les données relatives aux grandes structures du secteur tertiaire. Dans une deuxième étape, on a cherché à l'échelle des individus, des indications sur la fragmentation, les nouvelles interdépendances et la formation de nœuds. Cela a été réalisé à partir d'enquêtes sur le thème des préférences résidentielles, entre autres dans les gated et les pseudo-gated communities, ainsi que sur les espaces-vécus des habitants des zones résidentielles périurbaines. 


\section{Centre et périphérie des villes espagnoles}

7 La suburbanisation est en Espagne un phénomène relativement nouveau par rapport à l'Europe centrale. Il y a déjà eu dans la première moitié du $\mathrm{XX}^{\mathrm{e}}$ siècle les premiers déplacements vers l'extérieur qui, en règle générale, ne concernaient que les populations aisées des noyaux urbains, mais ceux-ci s'en sont tenus à des limites étroites et n'ont également touché que les grandes métropoles (voir Estébanez et Pérez 1990 pour Madrid). Si l'on ne tient pas compte de la formation à Barcelone, à Madrid et dans d'autres villes d'immigration de grandes cités résidentielles dans les années 60 et 70 (voir Estébanez 1990), la suburbanisation à plus grande échelle ne se produit que depuis les années 80 et plus particulièrement depuis l'essor économique qui a suivi l'adhésion de l'Espagne à l'Union européenne (1986). C'est également dans cette phase que se réalise une suburbanisation principalement sous forme compacte sur le plan architectural, notamment en bandes continues de maisons individuelles et en complexes d'appartements de plusieurs étages. Dans les plus grandes métropoles, les pavillons et les maisons jumelles s'étendent sur un périmètre plus étendu seulement depuis les années 90. Les cités et les habitations restent culturellement et majoritairement organisées dans des résidences plus compactes et le prix des terrains dans les périphéries des villes plus importantes favorise également la formation d'habitats en bandes continues, au lieu de plus grandes maisons individuelles, notamment en raison des revenus des ménages (toujours) plus faible qu'en Europe de l'ouest.

8 Cette suburbanisation galopante de rattrapage a désormais lieu parallèlement avec les nouveaux développements des zones périurbaines. Depuis les années 90 , cela aboutit à une migration massive vers la périphérie des pôles de développement et à un remodelage des périphéries urbano-rurales (Méndez 1994, Castro Martín 1998). Il en résulte le développement de nouveaux centres dans les périphéries, qui modifient la toute nouvelle ciudad dispersa (Monclús 1998). Est-ce que cela conduira vraiment à moyen terme à une ville réseau, c'est une question qui reste encore en suspens. En tous cas, et pour le moins à Madrid, on peut déjà percevoir les prémisses d'une edge city (Wehrhahn 2000 : 234); Tzschaschel (2004) parle également de nouveaux pôles dans les périphéries. Arroyo (2004) présente des résultats semblables pour la ville de Barcelone où il a pu démontrer l'existence d'un flux de personnes qui font la navette depuis déjà quelques décennies, ce qui a donné naissance à de nouveaux centres dans les périphéries. Dans la région métropolitaine de Barcelone, il s'agit de Vilafranca del Penedés, Sabadell, Terrassa, Granollers, Mataró et Vilanova i la Geltrú mais également et depuis peu, de Sant Celoni (Arroyo $2002: 16$ ).

9 Une particularité de la structuration et de la fonctionnalité des grandes métropoles espagnoles vient du fait que les agglomérations possèdent souvent un nombre extraordinaire de résidences secondaires. A la différence d'autres métropoles nationales ou mondiales, qui comptent un grand nombre de migrants pendulaires, ces résidences secondaires se trouvent non pas au cœur des villes, mais plutôt dans la zone périphérique. La raison en est la situation de beaucoup de grandes villes espagnoles en bord de mer ou, dans le cas de Madrid, à la montagne. Par conséquent, les périphéries de Barcelone, de Valence et de grandes villes du sud de l'Espagne sont saturées de résidences secondaires appartenant aux habitants des villes. À Madrid, l'attrait des communes de montagne résulte non seulement de la beauté du paysage, mais aussi du 
fait que la côte méditerranéenne est un peu trop éloignée pour des résidences secondaires, et c'est pour cela que beaucoup de madrilènes possèdent un appartement secondaire dans le nord et l'ouest de la région métropolitaine. On reviendra également sur ce phénomène.

Actuellement et parallèlement à la suburbanisation espagnole, on peut aussi observer une périurbanisation. Par rapport à la suburbanisation (voir définition dans Wehrhahn 2000 : 223), la périurbanisation signifie que :

- l'expansion de la zone de lotissement ne se rattache plus aux cités déjà bâties mais elle se trouve plutôt dans des îlots de l'espace rural;

- le lieu de travail se trouve à une distance bien plus éloignée que dans les cités classiques et suburbaines ;

- l'infrastructure d'approvisionnement est très déficitaire, à l'occasion de quoi se multiplient dans les centres des zones suburbaines non seulement les commerces et les offres de prestation de service, mais aussi les offres de services de loisir;

- la population y est aussi bien plus hétérogène, tant du point de vue démographique que si l'on se réfère au mode de vie et ;

- malgré une forte indépendance par rapport au centre-ville (qui en plus, joue un très grand rôle dans les villes européennes), il subsiste tout de même de fortes interdépendances à l'intérieur même des périphéries, mais aussi avec les centres-villes.

11 La périurbanisation n'est donc pas à confondre avec la counterurbanization dans le sens où l'entendent Fielding (1989) ou Champion et Vandermotten (1997) pour lesquels elle se rapporte à une déconcentration régionale (voir également Dézert 1991) ; ici, on fait donc allusion à une forme d'urbanisation régionale ou nationale des zones périphériques ${ }^{1}$.

12 Les centres des grandes métropoles espagnoles suivent donc d'un côté un processus semblable aux autres villes européennes qui subissent une dégradation de leurs architectures et infrastructures, une ségrégation sociale et ethnique et un vieillissement démographique. D'un autre côté, elles font toujours preuve d'une forte attraction pour le commerce de détail et font ainsi fonction comme auparavant, de centres culturels à l'intérieur des agglomérations et en cela elles diffèrent de beaucoup des métropoles d'Europe centrale et occidentale. Cela n'est pas seulement valable pour les grandes villes comme Barcelone, Valence ou Séville qui en plus possèdent une infrastructure très influencée par le tourisme; mais cela est valable bien plus encore pour les grandes villes moins importantes dont le centre reste comme avant le pôle le plus important pour la culture, les loisirs, les achats et la gastronomie. Dans quelle mesure et en raison de quels nouveaux développements des périphéries urbaines, peuton utiliser l'exemple de Madrid afin de définir une éventuelle transformation de la relation entre le noyau urbain et la périphérie. D'une part, il est vrai que Madrid représente une exception en raison de sa taille et de sa fonction de capitale. Mais, d'autre part, la ville de Madrid présente aussi de nouveaux développements de centres d'innovation représentatifs de ce processus d'urbanisation.

\section{Fragmentation et polarisation sociale à Madrid}

L'agglomération de Madrid (= Communidad Autónoma de Madrid/CAM) a démontré dans les 25 dernières années une dynamique de migration très grande et en même temps très hétérogène. A partir des années 50 et jusqu'aux années 70 , le district sud de 
la ville de Madrid s'est fortement agrandi à l'intérieur de la corona metropolitana (= zone suburbaine) (ex. Getafe) et les villes adjacentes Alcorón, Leganés et Móstoles (Estébanez 1990 : 15). Depuis le milieu des années 70, l'accroissement de la population s'est ainsi transférée sur la partie ouest de la corona metropolitan, entre autres sur Majadahonda, Pozuelo de Alarcón ou encore Las Rozas. Dans la zone suburbaine nord, quelques villes croissent rapidement comme San Sebastián de los Reyes et Alcobendas qui aujourd'hui comptent respectivement 61000 et 98000 habitants (données pour 2003; en comparaison avec 1970 : respectivement 25000 et 15000 habitants). Mais depuis les années 80, de plus petites communes comme Boadilla de Monte ou Villaviciosa de Odón enregistrent également un extrême accroissement de leur population.

L'évaluation des nouvelles données migratoires montre qu'un bouleversement important concernant le choix du lieu de résidence s'est effectué entre les années 80 et les années 90. Les mouvements migratoires ne convergent plus vers les communes suburbaines constituant un anneau à l'ouest et au sud du centre-ville mais ce sont désormais les municipalités qui se situent bien plus loin du centre-ville et un peu dans toutes les directions, jusqu'à $50 \mathrm{~km}$ du centre-ville de Madrid, au nord, à l'ouest et au sud-est qui sont les lieux d'accueil privilégiés des migrants (voir figure 1). Alors que dans les années 70 les cartes des totaux nets de migration permettent de mettre en évidence une suburbanisation classique (Wehrhahn 2000:227), on peut désormais observer une croissance en tâches, en particulier dans la zone la plus éloignée de la région métropolitaine. Les nouveaux immigrants ne viennent plus exclusivement du centre-ville comme dans la phase de suburbanisation, mais aujourd'hui, les immigrants de la zone périurbaine sont pour presque la moitié issus de la zone suburbaine. Vinuesa (2004: 40) évalue la part de cette migration intérieure à la zone périurbaine du centreville madrilène à $64 \%$ pour la période de 1991 à 2001. Pour la plupart, les migrants effectuent alors la deuxième étape d'une migration après qu'ils aient tout d'abord migré du centre de Madrid ou en tant que migrants intérieurs issus d'autres régions d'Espagne. Ils ont emménagé dans les blocs d'appartements des faubourgs du sud, de l'ouest et du nord.

Tableau 1 : Évolution de la population dans les différentes zones de la région métropolitaine de Madrid.

\begin{tabular}{|l|l|l|l|l|l|}
\hline Indicateur & Année & CAM & Municipio de Madrid & Zone suburbaine & Zone périurbaine \\
\hline Population & 1950 & 1.823 .410 & 1.553 .338 & 74.869 & 195.203 \\
\hline & 1970 & 3.761 .348 & 3.120 .941 & 413.337 & 227.070 \\
\hline & 1991 & 4.947 .555 & 3.019 .492 & 1.582 .077 & 354.986 \\
\hline & 1996 & 5.022 .289 & 2.866 .850 & 1.709 .956 & 445.483 \\
\hline & 2001 & 5.423 .384 & 2.938 .723 & 1.906 .360 & 578.301 \\
\hline Évolution (absolue) & $1970-75$ & 558.559 & 107.116 & 433.756 & 17.687 \\
\hline & $1975-81$ & 366.991 & -69.239 & 394.094 & 42.136 \\
\hline
\end{tabular}




\begin{tabular}{|l|l|l|l|l|l|}
\hline & $1981-86$ & 93.677 & -100.636 & 168.417 & 25.896 \\
\hline & $1986-91$ & 166.983 & -47.690 & 172.476 & 42.197 \\
\hline & $1991-96$ & 74.734 & -143.642 & 127.879 & 90.497 \\
\hline & $1996-01$ & 401.095 & 71.873 & 196.404 & 132.818 \\
\hline
\end{tabular}

Source : Census data et Zamora López 1993.

15 Ce développement de la croissance en périphérie, qui se déroule en même temps et de manière identique à un patchwork, correspond à une structure hétérogène des migrants similaire à ce que nous savons de la suburbanisation. Madrid se compose dans les années 70 et 80 principalement de jeunes adultes avec ou sans enfants. C'est ainsi qu'aujourd'hui la structure d'âge est très différenciée. En dehors des familles toujours les plus nombreuses, la zone périurbaine accueille également des personnes plus âgées. Ces personnes qui ont entre 45 et 60 ans sont toujours sur le marché du travail et il y a aussi des célibataires et de jeunes couples sans enfants. L'hétérogénéité croissante ne se manifeste pas seulement par la structure par âge. On observe également de fortes différences sociales à l'intérieur des mêmes classes d'âge: d'une part, il y a comme avant «les personnes âgées qui sont restées", malgré un processus sélectif d'émigration des périphéries lointaines vers les centres suburbains et le centre-ville ; d'autre part, ce groupe d'âge croît de manière très forte avec des personnes d'une autre origine sociale, qui souhaitent vieillir près des montagnes du nord et de l'ouest ; on les retrouve dans de petits appartements, mais aussi dans des villas de standing.

En outre, il y a un grand nombre de personnes, à la retraite ou presque, qui transforment leur résidence secondaire de la zone périurbaine en résidence principale (figure 1). Le nombre de résidences secondaires des périphéries éloignées des agglomérations était déjà très élevé depuis les années 80 (voir Pozo Rivera et Rodríguez Moya 1998 : 313). Cela évolue d'une manière constante et participe à l'accroissement du nombre de municipalités dans la zone suburbaine, où des appartements bon marché se transforment en domiciles de week-end. Ainsi, de 1991 à 2001, le nombre de résidences secondaires dans la zone suburbaine a augmenté de près de $120 \%$, soit 38225 , et dans la zone périurbaine (en partant d'une base bien plus élevée) de $32 \%$, soit 131288 (Leal Maldonado et Cortés Alcalá 2005: 32). Cela accroît encore la fragmentation sociodémographique et économique de l'espace périphérique. 
Figure 1 : Migrations nettes dans les municipalités de la région métropolitaine de Madrid de 2000 à 2002.

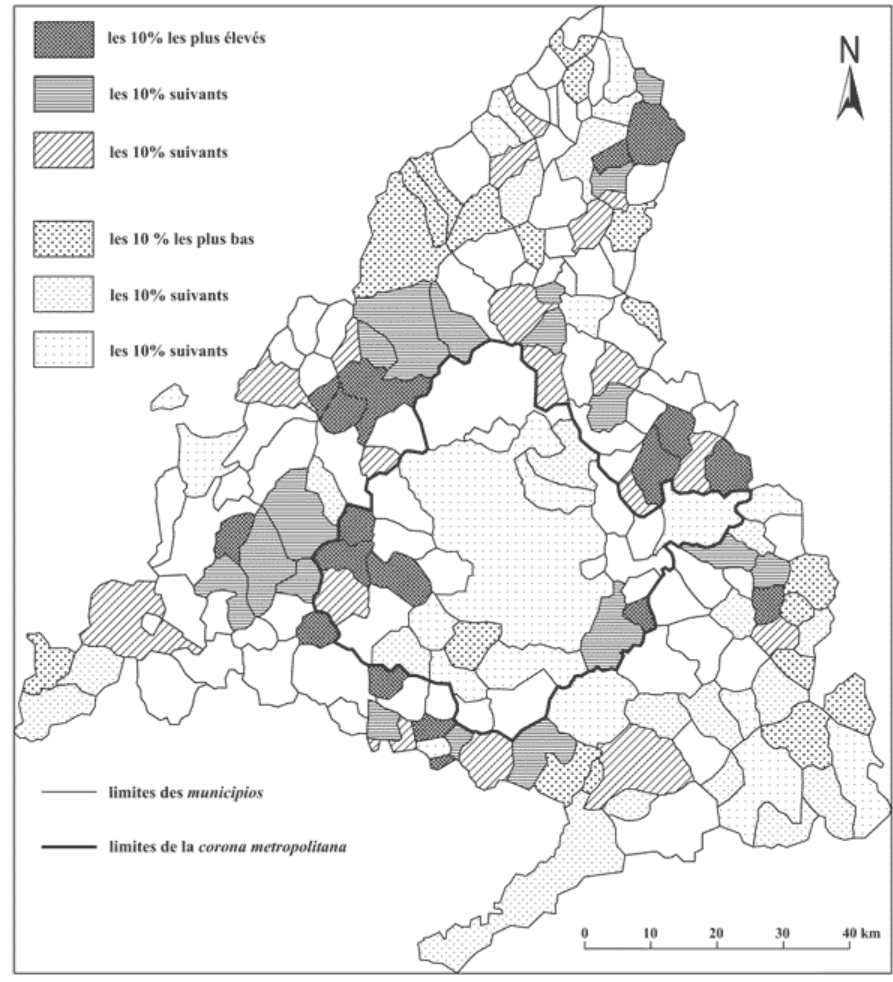

D'autres indices de la fragmentation des périphéries sont constitués par la répartition des structures de revenus (figure 3), des structures de formation et par la prise en compte des structures professionnelles. L'image de la répartition des revenus avec un cluster de revenu bien plus élevé et relativement indifférent à l'ouest et des revenus plus faibles dans le reste de la zone périphérique a peu à peu changé. Aussi, en fonction de ces indicateurs il faut retenir les fragments de municipalités avec des revenus moyens plus élevés dans la zone périurbaine. De plus, les extrêmes se trouvent en partie à proximité immédiate. 
Figure 2 : Résidences secondaires (en \% du total des résidences) dans les municipalités de la région en métropolitaine de Madrid en 2001.

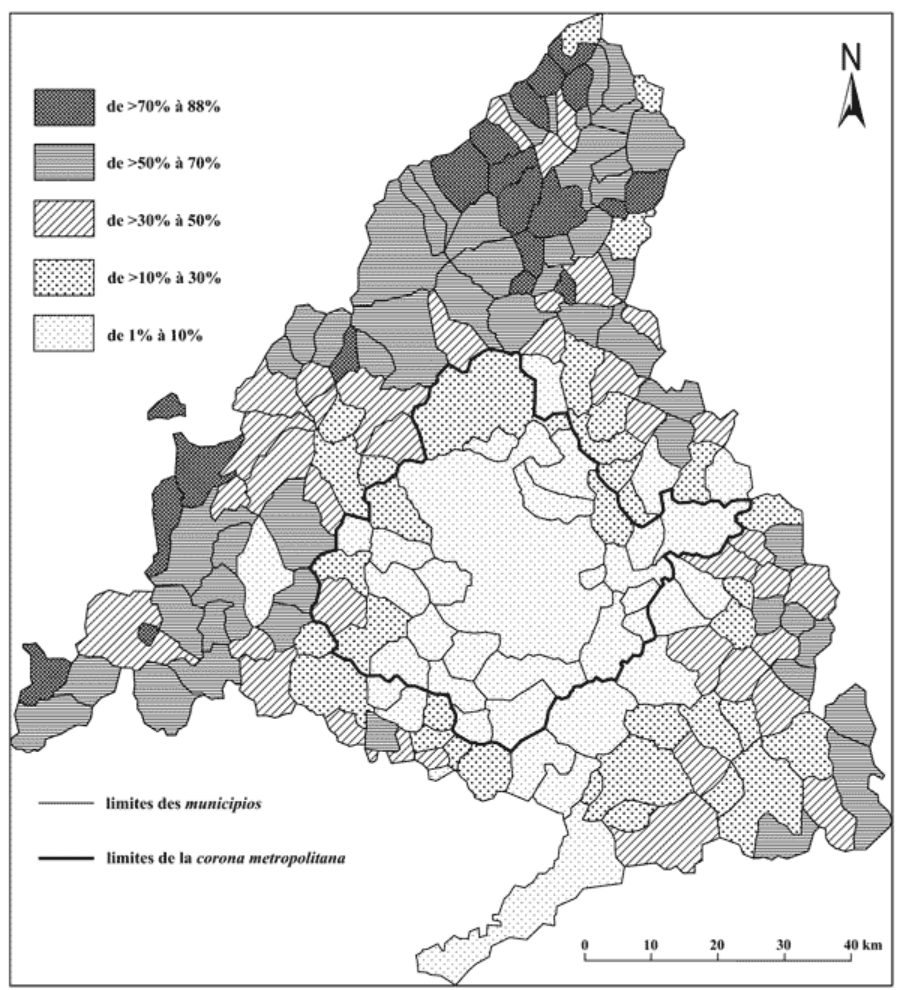

Figure 3 : Différenciation des revenus dans les municipalités de la région métropolitaine de Madrid en 2001 ('indice 100 représente la valeur moyenne pour la région urbaine de Madrid).

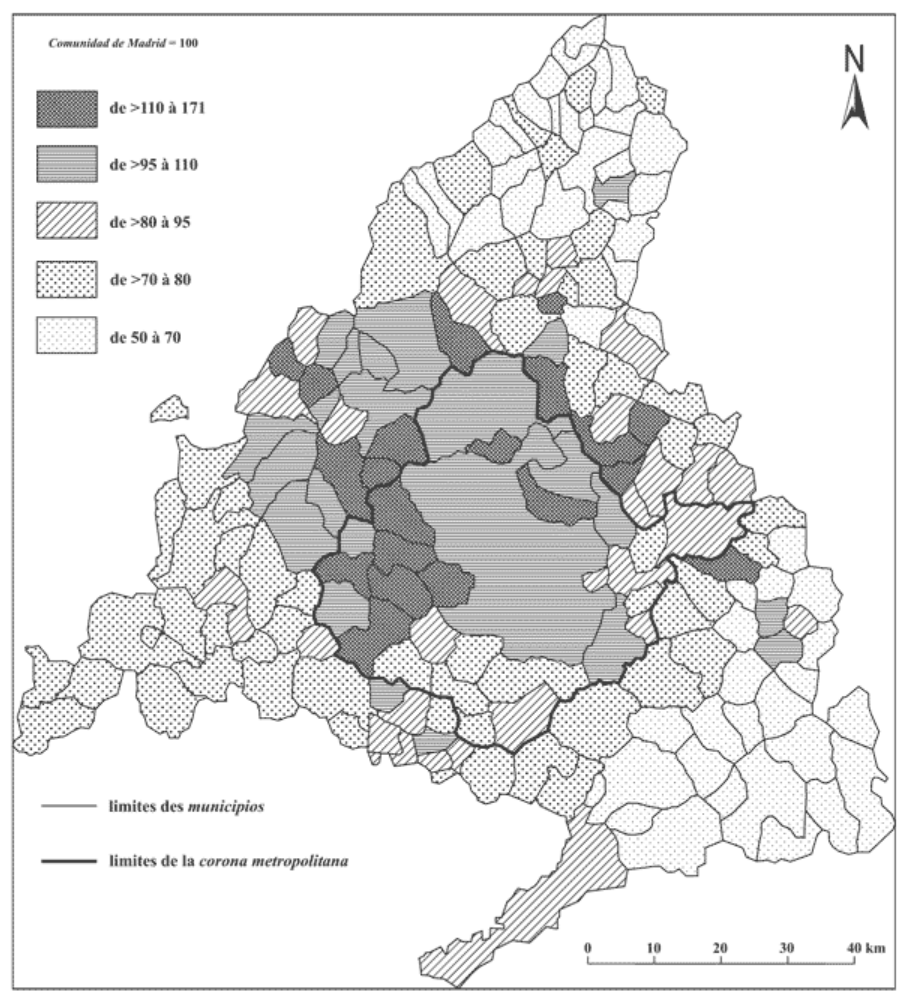



municipalités de la région métropolitaine. Sur la base des recensements de districts, il est possible de confirmer les différenciations suivantes, qui montrent clairement qu'une très forte polarisation sociale est également active à l'intérieur même du plus petit village. Ces différences de revenus reflètent la transformation de communes rurales de la périphérie qui sont affectées par une forte immigration d'un groupe de population avec de plus forts revenus. Dans des endroits comme Torrelaguna, Guadarrama, ou également dans de bien plus petits villages de la montagne avoisinante a lieu en ce moment une "gentrification de la zone rurale». Elle ne se marque pas seulement par des différences de revenus, mais aussi par les données concernant la formation et encore plus sur place, par des structures architecturales. Une transformation fonctionnelle du commerce de détail et de la prestation de services s'observe par le fait que de nouvelles offres de loisirs et de gastronomie s'implantent. Ces villages et ces petites villes offrent des contrastes flagrants avec les communes qui restent très imprégnées par la collectivité rurale traditionnelle, voire même, pour une certaine partie, affectées par l'abandon et la désertification.

19 La différenciation d'après le niveau d'instruction démontre un patchwork semblable (sans illustration; évaluation à partir de données de la Communidad Autónoma de Madrid 2001) : Certes, les proportions de personnes titulaires de diplômes de formation de plus haut niveau sont restées identiques dans la partie ouest de la corona metropolitana, mais entre temps, à l'intérieur même de la zone périurbaine, plusieurs municipalités se sont hissées dans le peloton de tête. C'est en règle général, parmi les communes qui ont connu une forte immigration dans les années précédentes, celles qui ont déjà beaucoup accueilli de personnes instruites, que se soit des personnes professionnellement actives ou non. Ces municipalités qui souvent se situent à la bordure des montagnes, se caractérisent par des structures socioéconomiques retardataires et contrastent d'autre part avec les communes avoisinantes. Vinuesa (2004: 62) analyse les structures d'instruction par zones de la Communidad Autónoma de Madrid ; il parvient au résultat que la zone périurbaine a atteint durant l'année 2001 pratiquement la même proportion de personnes titulaires d'un diplôme universitaire que la zone suburbaine. En ce qui concerne la répartition par catégories socioprofessionnelles titulaires d'un diplôme universitaire, les municipalités des périphéries obtiennent de meilleurs résultats que les suburbia en général (professions techniques, professeurs, etc.). Cependant, les catégories socioprofessionnelles des juristes, ingénieurs et autres personnes avec de bonnes formations professionnelles (et de bons salaires) continuent toutefois à résider de préférence dans la corona de l'ouest Pozuelo, Majadahonda, Villanueva, etc.

On peut donc retenir :

- qu'après un rattrapage accéléré de la suburbanisation des années 70 et 80 , une périphérisation de la croissance a lieu désormais à Madrid. Dans la zone périurbaine, la croissance s'effectue de manière isolée et non plus concentrée et plus ou moins globalement comme au temps de la suburbanisation ;

- les municipalités qui se trouvent dans la zone périurbaine dans un voisinage proche, présentent des profils socioéconomiques complètement différents. Une gentrification de la zone rurale proche des montagnes a également fait son apparition ; 
- de plus, le modèle de municipalités en croissance ou en décroissance est très hétérogène, tout spécialement au nord de la métropole où se situent très souvent ces deux types proches l'un de l'autre ;

- l'image doit être modifiée (et c'est déjà un facteur indicatif pour une hétérogénéité) par la présence de nombreuses résidences secondaires qui exercent en partie une forte influence sur la caractérisation socioéconomique des municipalités ;

- par contre, dans la zone suburbaine, on observe encore plus distinctement une différence à grande échelle entre les zones résidentielles aisées (à l'ouest) et les contrées plus pauvres (au sud). En raison du style de construction de bâtiments où prédominent les grandes cités de logements au sud et la construction de demeures familiales à l'ouest, cela ne changera pas dans les années à venir. Toutefois, et en raison de mesures de développement planifiées, le sud a connu ces dernières années une claire revalorisation, non seulement des structures d'entretien et de loisir mais aussi des structures de formation, à tel point que la corona sur ne peut plus seulement être qualifiée de « ville dortoir pour la population plus pauvre ».

\section{Gated et pseudo-gated communities en tant qu'expression définissant une façon d'habiter postmoderne?}

21 La question de la fragmentation économique est étroitement liée à l'expansion de nouvelles façons d'habiter qui, dans le contexte théorique de l'urbanisation postmoderne dans le sens où Soja (2000) l'entend ${ }^{2}$, a des rapports avec la prolifération de gated (et pseudo-gated) communities. Les gated communities ne se caractérisent pas seulement par leur isolement, mais aussi par le degré d'isolement vis-à-vis de ceux qui n'y habitent pas. Elles reflètent également les différents groupes sociaux par rapport à leurs besoins concernant les habitations et les lotissements.

Si l'on prend Madrid comme exemple, il faut également prendre en compte, dans le cadre d'une analyse théorique, aussi bien le contexte structurel (les facteurs politiques, économiques, socio-culturels sous l'influence de conditions globales et locales) que, sur un plan individuel, les motivations du choix du lieu d'habitation dans une gated ou pseudo-gated community, ainsi que les acteurs économiques et politiques locaux (voir Wehrhahn 2003 : 303). Les besoins en habitations et leurs conséquences sur les espaces ne doivent pas seulement être considérés en tant que théorie d'action, mais une explication valable ne s'imposera pas sans prendre en considération le contexte. Cette contribution porte néanmoins principalement sur les motivations individuelles ${ }^{3}$ et ne reprend pas la typologie des gated et pseudo-gated communities à Madrid, qui est déjà présentée dans d'autres publications. Il sera donc question dans ce qui suit, de définir le rôle des besoins en logement de la société (post) urbaine qui influence l'apparition de ces formes de cités.

Des analyses détaillées des motivations de ceux qui viennent de s'installer dans ces cités madrilènes montrent qu'il n'existe pas une motivation simple et exclusive ayant une valeur explicative suffisante. C'est le cas par exemple de la sécurité qui est souvent citée, comme dans d'autres pays, comme raison déterminante pour une prolifération des gated communities (voir Jürgens et Landmann 2005 pour l'Afrique du Sud ou Caldeira 1992 pour le Brésil). Bien plus encore, il s'agit de réfléchir d'abord sur un deuxième aspect essentiel : à Madrid, le prestige étroitement associé à de nouveaux styles de vie, joue un rôle également aussi important que la présence de services de garde. Il faut 
aussi prendre en considération le fait que la sécurité est interprétée de manière souvent différente dans les sondages. Dans le cas des recherches faites à Madrid, deux style de "sécurités » différentes ont pu être déterminés : il y a d'abord la sécurité ordinaire, c'est-à-dire le fait de ne pas être dérangé, la tranquillité à l'égard du passage des voitures, la « sécurité » pour les enfants qui jouent dans la rue, le fait de ne pas être importuné par des mendiants ou des distributeurs de prospectus. La deuxième forme de sécurité entend un contrôle d'entrée efficient, la protection réelle contre la criminalité et par conséquent un isolement efficace du monde extérieur. Cette dernière n'est réellement garantie que dans peu de gated communities de Madrid, par exemple à Calme House (municipalité de Boadilla del Monte) ou à Ciudad Santo Domingo (municipalité de Algete). La sécurité est par conséquent interprétée en tant que sécurité ordinaire «molle » et c'est ainsi qu'elle est qualifiée par les habitants de ces communities, dans des enquêtes qualitatives. La sécurité a premièrement pour fonction le fait que l'on puisse vraiment vivre plus au calme dans sa cité résidentielle, être moins importuné et avoir un sentiment de sécurité plus élevé pour les enfants. Deuxièmement, la "sécurité » doit aussi être interprétée en tant que facteur dissimulé d'un certain prestige, car entre temps, le fait d'habiter dans des résidences closes constitue également un symbole pour le statut. D'après les enquêtes, les frontières entre les prétendus établissements de sécurité, contrôles, portails d'entrée, domaines d'entrée soignés et les installations vertes se mélangent et doivent donc être interprétés ensemble. Dans ce cas, il est clair qu'il est impossible de donner un pourcentage précis. A partir d'autres interviews qualitatives effectuées auprès des habitants, des gérants et des surveillants de gated et pseudo-gated communities dans Madrid, on peut ainsi formuler l'hypothèse que les mesures de sécurité augmentent également le prestige des cités. D’après Soja (2000), le retranchement et par conséquent, la naissance de carceral cities, peut être également interprété comme l'expression d'un style de vie, un style de vie de la séparation et en même temps de la distinction au moyen d'établissements fortifiés.

24 Si l'on va plus loin, la question se pose de savoir quelle signification ont les portes, les clôtures et les murs, qui sont en effet physiquement présents, mais qui cependant ne remplissent pas une fonction d'isolement, car ils restent tout le temps ouvert et sont relativement perméables (du moins la journée). Cette sorte de porte symbolique est très présente dans la périphérie de Madrid. Ces symboles ouvrent aux visiteurs le chemin des pseudo-gated communities et ils représentent en même temps une fermeture et une vie privée qui, quelque motivation qu'elle ait (légales, financières, etc.), ne peut pas faire juridiquement l'objet de poursuites. Les portails symboliques marquent des territoires; ils indiquent aux personnes qui n'appartiennent pas à cette enclave que leur présence n'est pas souhaitée derrière ces portes, panneaux indicateurs ou barrières ouvertes. Les habitants des pseudo-gated communities ont mis de manière absolument consciente ces portes symboliques qui agissent comme caractère distinctif afin d'accentuer leur communauté propre. C'est particulièrement clair dans des pseudogated communities comme El Coto (dans la périphérie ouest de Madrid, donnant déjà sur la province de Guadalajarra), où n'exerce pratiquement aucune société privée de surveillance, mais (jusqu'en 2003) où tout de même des barrières et des cabanons de surveillance indiquent que l'on entre sur un territoire étranger. Lors des enquêtes de 1999 et de 2000 (de l'auteur), on a posé la question de savoir quelles motivations principales avaient les personnes qui choisissaient ces endroits comme résidence; les réponses renvoyaient souvent à l'importance des installations de sécurité de ces cités, 
bien qu'il ne soit presque pas possible de juger cela plausible, tant par rapport à la protection à l'encontre de la criminalité que de celui de "vivre en paix et sans être dérangé ». Les places vertes et les portails d'entrée ont été pareillement définis comme critère de choix pour un emménagement, bien que ces deux composantes ne soient pas totalement louables dans le cas d'El Coto. Les installations de sécurité et les éléments particuliers de paysages et de jardins ont une fonction à caractère distinctif. Dans cette mesure, ce symbole remplit bien la raison de documenter cette séparation. Des questionnaires ont apporté des résultats similaires pour de plus petits pâtés de maisons individuelles à Las Rozas, qui également ne dispose que d'installations symboliques de sécurité pour les piétons ${ }^{4}$.

Les gated et pseudo-gated communities sont à voir de manière générale comme l'expression d'un nouveau style de vie postmoderne. La composition d'autres groupes d'habitants ou plutôt d'agglomérations se tient au premier plan, ce que montre par exemple la construction de gated communities à l'intérieur même de plus grosses gated et pseudo-gated communities. Ainsi à El Encinar de los Reyes ou à Boadilla del Monte, beaucoup de petites communautés fermées se trouvent à l'intérieur d'une pseudo-gated community. C'est l'exemple de Calme House à l'intérieur de Valdepastores/Boadilla del Monte.

\section{Fragments et nouveaux nœuds du secteur tertiaire dans la périphérie}

La restructuration de la périphérie madrilène ne s'est pas seulement effectuée comme une suite logique de la suburbanisation et de la périurbanisation de la population. Depuis le milieu des années 80 , des entrepreneurs du secteur tertiaire et en particulier du commerce de détail se sont également établis en nombre croissant, aussi bien dans la corona metropolitana que dans des communes bien plus éloignées. Dans une première phase de ce processus, on a d'abord construit des centres commerciaux de tailles différentes. De 1985 à 1999 leur nombre passe de 83 à 104 dans la CAM (3 seulement sont bâtis dans la ville même) La superficie des espaces de vente a été multipliée par 7 (Guia de grandes superficies, Comunidad de Madrid, diverses années). A lui seul, le nombre des hypermarchésest passé de 17 à 53 entre 1992 et 2000 (Kleinefenn 2003).

Ces établissements commerciaux se concentrent aujoud'hui sur plusieurs domaines (figure 4): d'une part au sud-ouest de la corona metropolitana et en particulier à Alcorcón où se trouve le deuxième plus gros centre commercial de Madrid, le Parque Sur, avec une superficie de commerces d'environ $120000 \mathrm{~m}^{2}$. La concentration d'établissements commerciaux de vente au détail comme celle de commerces de gros, ainsi que le taux élevé d'industries de loisir s'expliquent du fait que près de 800000 personnes réparties sur plusieurs grandes villes vivent dans ce secteur et à cela viennent s'ajouter plusieurs centaines de milliers d'habitants de la circonscription sud du noyau urbain madrilène. Le programme de développement pour la dite Gran Sur, qui prévoyait une amélioration globale des conditions de vie dans cette zone de vieille industrie en partie dégradée, ainsi que dans celle des extensions urbaines des années 60 et 70 a déjà été mis en place avec succès. En tant que société régionale de développement, l'ARPEGIO se présente comme l'acteur principal de ce développement qui est responsable d'une grande partie des mesures prises. Le Gran Sur qui se trouve sur le chemin de la modernisation, ou plus particulièrement de la post modernisation, 
ne doit cependant pas être dans sa totalité caractérisé comme un nouveau maillon de la croissance, mais plutôt comme une zone de lotissements pour les travailleurs et un pôle industriel qui grandit depuis plusieurs décennies.

Figure 4 : Structure des cités et pôles du secteur tertiaire dans la région métropolitaine de Madrid.

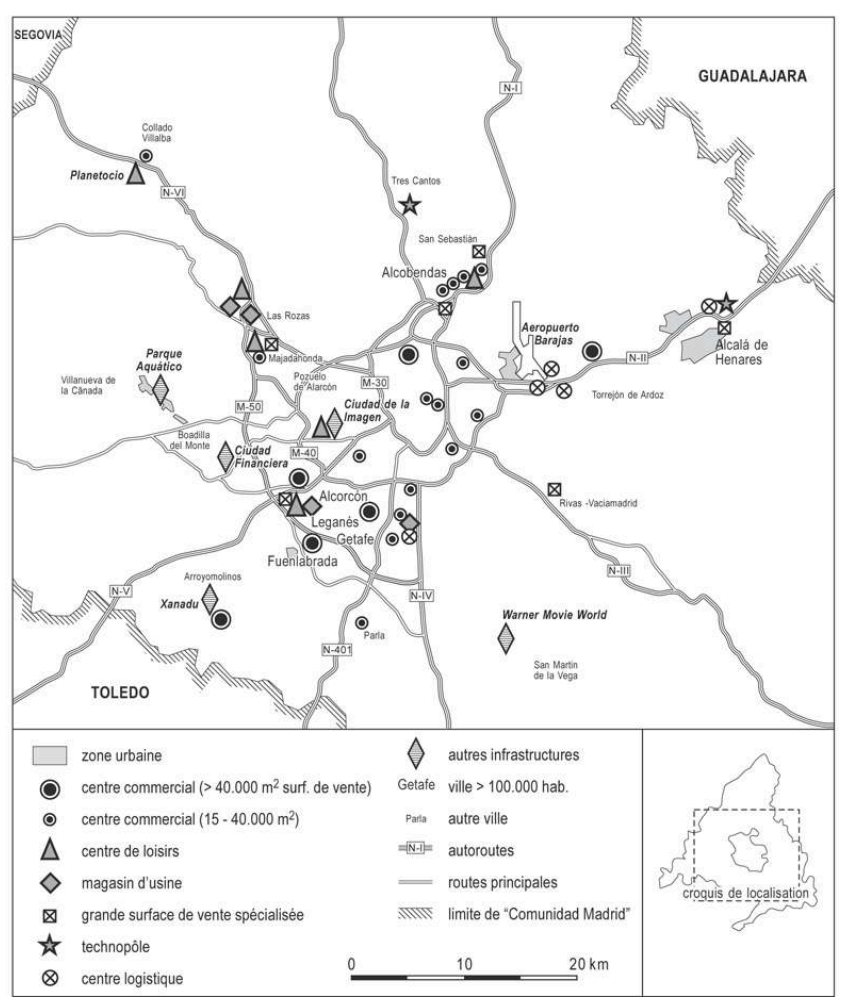

À présent, il reste à définir un autre pôle, une deuxième concentration qui s'est développée dans la périphérie nord d'Alcobendas et de San Sebastián de los Reyes. Cette zone présente un clair excédent de postes de travail. De nouvelles grandes implantations du secteur tertiaire ainsi que des zones industrielles se situent pour la plupart dans la municipalité d'Alcobendas, le long de l'autoroute NI en direction de Burgos. Ainsi par exemple, une importante filiale d'Ikea s'y est également installée ; Alcobendas est aujourd'hui l'endroit en dehors du noyau urbain de Madrid où il y a le plus de bureaux. Le nombre de personnes qui y viennent pour travailler est particulièrement important. Il s'élève à $70 \%$ sur un total de 55000 emplois (Vinuesa 2004: 106). Ce pôle est le plus important centre d'approvisionnement extérieur du noyau urbain, non seulement pour les municipalités avoisinantes, mais également pour toutes les communes plus éloignées dans le nord et le nord-est jusqu'aux frontières avec la province de Guadalajarra.

Actuellement, un nouveau pôle aussi important est en train de se former dans l'ouest : Las Rozas, Pozuelo de Alarcón et Majadahonda. Ces trois villes avec chacune entre 40000 et 76000 habitants ont une fonction de centre d'attraction pour les banlieusards des environs plus ou moins proches. Vinuesa (2004: 106-7) donne des chiffres de l'emploi qui sont respectivement de 27000 et 18000 pour Las Rozas - Pozuelo et pour Majadohonda et il évalue les pourcentages de banlieusards à $70 \%, 67 \%$ et $66 \%$ pour les trois municipalités. Des communes plus petites comme Boadilla del Monte et Villaviciosa de Odón présentent également de forts pourcentages de banlieusards (67 \% 
et $65 \%$ avec pour chacune 8000 emplois). Ces communes forment un nouveau centre du développement économique de l'agglomération et réunissent sur leur territoire une grande partie des entreprises basées sur l'innovation et la technologie qui auparavant étaient installées à Madrid. De plus, de nouvelles infrastructures du secteur tertiaire s'y sont implantées : des centres commerciaux et des centres de loisirs (Heron City à Las Rozas), deux factory outlet centers ainsi que la cité audiovisuelle Ciudad de la Imagen à Pozuelo. Avec ses interconnections entre les multiples infrastructures tertiaires en lien avec de nouveaux projets résidentiels de grande envergure comme Parque Empresarial Las Rozas, le nœud de Las Rozas peut être considéré, en 2005, comme une sorte de edge city américaine (Garreau 1991), avec cependant quelques modifications européennes en ce qui concerne l'éloignement des centres urbains.

En outre, en 2004, un autre projet important sans équivalent en Europe a été réalisé dans cette zone : Bodilla del Monte a vu s'installer dans sa périphérie une véritable ville bancaire («Ciudad Financiera») et par ailleurs, si l'on compare avec d'autres municipalités de Madrid, il s'agit de la commune qui s'est le plus développée au cours de ces 20 dernières années (2004: environ 30000 habitants). Sur une superficie de $165 \mathrm{ha}$, elle comprend 13 immeubles avec $100000 \mathrm{~m}^{2}$ de bureaux pour le promoteur du projet, la Banco Santander Central Hispanico $(\mathrm{SCH})$, mais aussi des entrepreneurs orientés vers la prestation de services, un hôtel, un centre de santé, une bibliothèque, cinq restaurants, un golf 18 trous, un centre de fitness, une piscine, un terrain de tennis et un de squash, ainsi que d'autres détaillants et établissements sportifs. Des établissements communautaires (crèches, etc.) sont également intégrés (Reuters 05.04.2004). Dans la première phase, près de 5000 emplois ont été créés, mais ils ne sont cependant pas "nouveaux » puisqu'ils résultent simplement de la réunion de plus de 16 anciens immeubles de la BSCH de l'agglomération de Madrid. Un agrandissement de la surface de bureaux est planifié, afin de créer encore 3000 à 5000 nouveaux emplois.

31 Bien que cette « ville bancaire » soit définie par la presse espagnole comme un projet unique en Europe, l'idée n'est pas tout à fait nouvelle. On peut citer comme exemple la ville Siemens à Berlin comme étant l'intégration de regroupements d'entrepreneurs avec des aménagements d'infrastructures de styles très différents et de l'habitat, qui n'y est cependant pas pris en compte, en raison de l'opposition du côté des syndicats et des autorités politiques locales. Constituant un nouveau fragment isolé du secteur financier situé très loin des extensions urbaines, il se pose bien entendu une question intéressante qui est de savoir si cette cité qui rassemble des entreprises dans une situation d'isolement spatial par rapport au reste du secteur financier de Madrid, peut retenir ce "district financier" dans sa zone, ou si au contraire cela va amener à d'autres transferts d'activités financières vers Boadilla del Monte. Le transfert vers la périphérie a en tout cas l'air d'être financièrement rentable : les coûts de construction d'environ 480 Mil. $€$ seront compensés dans peu d'années par la vente aux particuliers de 16 lotissements situés au centre, ainsi que par les économies d'échelle réalisées par le regroupement (Reuters 05.04.2004).

À côté de la formation de nouveaux centres dans la zone périphérique de Madrid, on observe également une fragmentation qui va dans le sens d'une urbanisation postmoderne. La Ciudad Financiera, ainsi que beaucoup de centres de marchés spécialisés dans des situations non intégrées, en sont la preuve, tout comme les grands centres commerciaux espagnols et les centres de loisirs Xanadu dans la municipalité 
d'Arroyomolinos ou le Warner Movie World à San Martín de la Vega (voir figure 4). Xanadu se compose de plus de $200000 \mathrm{~m}^{2}$ de vastes magasins et de restaurants avec plusieurs point d'ancrage, un circuit de kart et comme plus gros point d'ancrage, une piste de ski de $250 \mathrm{~m}$. L'établissement a été ouvert en 2004 et ne compte pas seulement comme étant le centre commercial et de loisirs le plus grand d'Espagne, mais aussi et surtout le plus moderne. Le Warner Movie World qui lui aussi n'est pas intégré et qui possède ses propres infrastructures de connexion sous forme de voies express au sud de Madrid, a déjà été ouvert en 2002.

\section{Conclusion}

Comment les développements urbains présentés ici doivent-ils être interprétés? Afin d'expliquer les processus démesurés à l'échelle européenne, il faut d'abord mentionner la puissance de la restructuration économique dans ce pays qui présente un besoin de rattrapage après pratiquement 40 années de dictature et par conséquent un fort isolement vis-à-vis du reste de l'Europe, mais un pays qui a également su profiter de l'affluence des subventions européennes (jusque dans la région de la capitale!). Ces conditions économiques peuvent être complétées par d'autres réflexions sur les structures: après le passage à la démocratie, la société s'est transformée et l'amélioration du niveau des revenus contribue également à ce que les besoins en matière de logements et les souhaits de style de vie puissent être satisfaits. Le contexte politique de planification était dans les années 80 également dans une phase de défrichage et rendit possible ou plutôt favorisa les constructions dans la périphérie même, de bâtiments non planifiés (Canossa 2000). Dans la région de Madrid subsistent aujourd'hui encore des contraintes concernant les autorisations de projets de développement dans les municipalités de la périphérie. Ces contraintes s'exercent à travers les autorités de planification régionale, bien qu'un plan régional pour la Communidad de Madrid n'existe toujours pas, alors que c'est exigé par la loi.

Il y a, au niveau des acteurs, de multiple motivations: les acteurs économiques de l'aménagement de la ville, comme les développeurs et les entrepreneurs immobiliers, peuvent réaliser des concepts de cités et des développements industriels différents. Au niveau de la municipalité, les acteurs politiques peuvent exercer leur influence sur les différentes sortes de développement des localités, dans le sens où ils exploitent leur champ de négociation de diverses manières lorsqu'il s'agit de lotissements. Les acteurs privés se décident pour différentes formes de résidence et d'emplacements commerciaux ou de loisirs à l'intérieur même de l'agglomération en fonction de leur style de vie, des phases de leur cycle de vie et de leurs revenus. Les styles de vie seront réalisés dans les cités isolées de lotissement périurbain sous forme de portes symboliques. Ces cités peuvent cependant amener à de fortes imbrications entre la gated community et la rue commerçante principale (Serrano) dans le district de Salamanca au centre de Madrid, comme par exemple, le prêt-à-porter qui se vend et s'achète seulement dans ces boutiques (Wehrhahn 2001). D'autre part, de nouveaux magasins et des lieux de divertissement attirant particulièrement les jeunes apparaissent dans les centres commerciaux de la périphérie (Gutiérez et al. 2001). Un autre processus qui s'avère être bien présent à Madrid, consiste à faire le lien entre les styles de vie et les activités de loisirs, ce qui se manifeste entre autres par la gentrification dans les espaces ruraux et des villages remodelés pour le tourisme sous la 
forme de villages d'artistes ou encore de villages musées (ex. Patones de Arriba). On ne peut ici que donner un aperçu qualitatif de ces développements, étant donné que les recherches à ce sujet ne sont pas encore achevées. Les rôles que jouent les facteurs globaux et/ou locaux dans ces processus qui se déroulent à Madrid de manière particulièrement rapide et intense, restent de même sans réponse.

Ainsi, l'hétérogénéité et la fragmentation de la périphérie sont de manière générale à voir en tant que résultats des conditions structurelles et de leurs modifications. D'un autre côté, elles dépendent également des décisions d'acteurs et de groupes d'acteurs très hétérogènes. De plus, des centres sont apparus récemment qui, à long terme, conduisent à des connexions complètement nouvelles dans la zone suburbaine et périurbaine, comme Gutiérrez (2003) le montre avec l'exemple des relations des banlieusards. Qu'à court ou à long terme, une ville réseau apparaisse et que l'urban sprawl en tant que concept de la structure des espaces soit ainsi démenti ; cela reste une question ouverte. Une "dissolution du noyau urbain» n'est pas pensable pour l'agglomération de Madrid eu égard à l'importance que peut encore avoir la centralité de ce noyau.

\section{BIBLIOGRAPHIE}

ARING J. (1999). - Suburbia - Postsuburbia - Zwischenstadt. ARL Materialien, p. 262, Hannover. ANGLADE F. (1992). - « Le périurbain à La Rochelle : utilisation desméthodes de l'analyse des données ». Norois 39, p. 229-240.

ARROYO M. (2002). - «El Mercado de Trabajo y la Reestructuracióndelas Áreas Metropolitanas ». In : Scripta Nova. Revista Electrónica de Geografía y Ciençias Sociales, 119 (60).

BORSDORF A. \& ZEMBRI P. (eds.) (2004). - Structures. European Cities Insights on Outskirts. Cost Action C10. Bruxelles.

BURDACK J. (2005). - « Die metropolitane Peripherie zwischensuburbanen und postsuburbanen Entwicklungen. Diskurse und Methodik der Untersuchung ». In : BURDACK J., HERFERT G. \& RUDOLPH M. (eds.).Europäische metropolitane Peripherien. BRG 61. Leipzig, p. 8-23.

CALDEIRA M.T. (1992). - City of Walls : Crime, Segregation, and Citizenship in São Paulo. Berkely.

CANOSA E. (1995). - La promoción inmobiliaria en la periferia noreste de Madrid. Madrid (Universidad Autónoma de Madrid).

CASTRO MARTÍN T. (1998). - Informe monográfico del tomo I de la estadística de la población de la Comunidad de Madrid 1996, Madrid.

CHAMPION T. \& VANDERMOTTEN C. (1997). — « Migration,Counterurbanization and Regional Restructuring in Europe. »In : BLOTEVoGEL H.H. \& FIELDING A.J. (eds.) - People, Jobs andMobility in the New Europe, Chichester,p. 69-90.

DAVIS M. (1990). - City of quartz : Excavating the future in Los Angeles. London, New York. 
DEAR M. (2000). - The postmodern urban condition. Malden, Mass.

DEAR M. \& FLUSTY S. (1998). - « Postmodern Urbanism ». Annals ofthe Association of American Geographers 88, 1, p. 50-72.

DEMATTEIS G. (1998). — « Suburbanización y Periurbanización. Ciudades Anglosajonas y Ciudades Latinas ». In : Monclús F. J. (ed.) - La Ciudad Dispersa, Barcelona, p. 17-33.

DÉZERT B. (1991). - « Causes et conséquences géographiques de lamétropolisation et de l'exurbanisation ». In : Bulletin de l'Association de Géographes Français 68, p. 133-138.

DÍAZ ORUETA F. (1991). - Los Procesos de Descentralización Demográfica en las Grandes Ciudades : El Caso Español y una Aproximación a otras Ciudades del Sur de Europa. In : Economía y Sociedad 5, p. 221-233. DUBoIS-TAINE G. (ed.) (2004). — « From Helsinki to Nikosia. ElevenCase Studies \& Synthesis. European Cities Insights on Outskirts ». Cost Action C10, Bruxelles.

ESTÉBANEZ ÁlVAREZ J. (1990). - « Génesis del modelo territorialmetropolitano madrileño ». In : eSTÉBANEZ ÁlVAREZ J. (ed.). - Madrid, Presente y Futuro. Madrid, p. 9-40.

ESTÉBANEZ ÁlVAREZ J. \& Pérez C. (1990). - « La crisis del sistemametropolitana desarrollista ». In : ESTÉBANEZ ÁlVAREZ J. (ed.). - Madrid, Presente y Futuro. Madrid, p. 211-262.

FIELDING A. J. (1989). - « Migration and Urbanization in WesternEurope since 1950 ». In : The Geographical Journal 155, p. 60-69.

FRANZÉN M. \& HALLEUX J.-M. (2004) (eds.). - Dynamics. EuropeanCities Insights on Outskirts. Cost Action C10. Bruxelles.

GARREAU J. (1991). - Edge cities. Life on the New Frontier. New York.

GUTIÉRREZ J. (2003). - Nuevas periferías metropolitanas : transformaciones territoriales y cambios en la movilidad. El caso de Madrid. Madrid (Universidad Complutense).

GUTIÉRREZ J., CARRERA M.C., CHICHARRO E., KLEINEFENN A., WEHRHAHN R. (2001). - « El perfil de los consumidores en los grandes centros comerciales y de ocio de la periferia de Madrid ». In : Boletín de la Asociación de Geógrafos Españoles 31, p. 61-85.

HALL T. (1998). - Urban Geography. London.

JÜRGENS U., LANDMANN K. (2005). - « Gated Communities in SouthAfrika ». In : GLASZE G., WEBSTER C., FRANTZ, K. (eds.). - Private Cities. Global and Local Perspectives. London, New York, p. 109-126.

LEAL MALDONADO J. \& CORTÉS ALCALÁ L. (2005). - « Características del parque de viviendas de la Comunidad de Madrid según el Ceso de2001 ». Estudios y Analysis (Comunidad de Madrid), Madrid. LÓPEZ DE LUCIO R. (1998). - « La incipiente configuración de unaregión urbana dispersa : el caso de la Comunidad Autónoma deMadrid (1960-1993) ». In : Monclús J. (ed.). - La ciudad dispersa, Barcelona,p. 169-196.

MCELDOWNEY M. (ed.) (2004). - Governance. European Cities Insights on Outskirts. Cost Action C10. Brüssel.

MÉNDEZ R. (1994). - « Crecimiento Periférico y Reorganización delModelo Metropolitano en la Comunidad de Madrid ». In : Economía y Sociedad, 10, p. 149-173.

MÉNDEZ R. (2001). - « Transformaciones económicas y reorganizaciónterritorial en la región metropolitana de Madrid ». Revista eure, 27 ; 80 ; p. 141-161.

MONCLÚS J. (ed.) (1998). - La Ciudad dispersa. Barcelona. 
POZO RIVERA E. \& RODRÍGUEZ MOYA J. M. (1998). - « La Evoluciónde la Población en la Comunidad de Madrid (1991-1996) ».In : Anales de Geografía de la Universidad Complutense 18, Madrid, p. 299-316. ROYUELA MORA V., VAYÁ VALCARE E., SURI“ACH I CARALT J. (2003). — « L'Elecció de Destinacions Residencials no Forçades aL'Entorn Metropolità de Barcelona. Es Cas de Sant Cugat Del Vallès ». In : Scripta Nova. Revista Electrónica de Geografía y Ciençias Sociales, 7, 146.

SIEVERTS T. (1998). - Zwischenstadt zwischen Ort und Welt, Raum und Zeit, Stadt und Land. Bauwelt Fundamente 118. Braunschweig, Wiesbaden.

SOJA E.W. (1995). - « Postmodern Urbanization. The SixRestructurings of Los Angeles ». In : WATSON S. \& GIBSON K. (eds.). - Postmodern Cities and Spaces, Oxford, p. 125-137.

SOJA E.W. (2000). - Postmetropolis. Critical Studies of Cities and Regions. Oxford.

SOUMAGNE J., BOULY A. C., FOURNIER I (1992). — «Changements périurbains autour de Poitiers ». Norois 39,154, p. 241-248.

TZSCHASCHEL S. (2005). — « Madrid ». In : BURDACK J., HERFERT G., RUDOLPH M. (eds.). - Europäische metropolitane Peripherien. BRG 61, Leipzig, p. 61-81.

VINUESA J. (1994). - La Población de Madrid. Análise de la Dinámica Demográfica de la Región 1981-1991. Madrid.

VINUESA J. (2004). - Características demográficas de la Comunidad de Madrid según el Censo de 2001. Estudios y Analysis (Comunidad de Madrid), Madrid.

WEHRHAHN R. (2000). - « Zur Peripherie postmoderner Metropolen :Periurbanisierung, Fragmentierung und Polarisierung, untersucht amBeispiel Madrid ». In : Erdkunde, 54, p. 221-238. WEHRHAHN R. (2003). — « Postmetropolis in Spanien? NeueEntwicklungen in Madrid und Barcelona ». In : GeographischeRundschau, 55, 5, p. 22-28.

WEHRHAHN R. \& RAPOSO R. (2005). — « The Rise of gated residentialNeighbourhoods in Portugal and Spain : Lisbon and Madrid ». In : GLASZE G., WEBSTER C., FRANTZ K. (eds.) (2005). - « Private Cities ». Global and Local Perspectives. London, New York, p. 170-189.

WOOD G. (2003). - Die Wahrnehmung städtischen Wandels in der Postmoderne. Untersucht am Beispiel der Stadt Oberhausen. Stadtforschung aktuell 88. Opladen.

ZAMORA LÓPEZ F. (1993). - Las migraciones de la Comunidad de Madrid de 1960 a 1989. Madrid.

\section{NOTES}

1. Le terme de "périurbanisation" n'est bien entendu pas souvent employé de manière uniforme (voir Anglade 1992 ou Soumagne et al. 1992 qui avec leur définition se rabattent plutôt sur une counterurbanization).

2. Voir Wehrhahn 2003 ainsi que Wehrhahn et Raposo 2005 pour une interprétation plus vaste de l'apparition et de la prolifération de gated communities à Madrid.

3. Voir Wehrhahn 2003 ainsi que Wehrhahn et Raposo 2005 pour une interprétation plus vaste de l'apparition et de la prolifération de gated communities à Madrid.

4. Une carte à puce est nécessaire afin d'ouvrir les portes et de laisser passer les véhicules personnels dans cette rue où sont construites de chaque côté des résidences individuelles. 


\section{RÉSUMÉS}

À Madrid, les processus de la post-suburbanisation s'observent sous beaucoup de formes. La restructuration économique après la fin de l'ère Franco, l'entrée dans l'Union européenne et les changements économiques et sociaux qui ont suivi, mais aussi la dissolution des conceptions plus strictes de l'aménagement du territoire qui prévalaient jusqu'alors par des processus plus libéraux ont changé la ville à un rythme sans précédent. Ces changements qui ont affecté l'ensemble de l'organisme urbain sont particulièrement marqués dans les anciennes périphéries rurales. La ségrégation sociale et la fragmentation urbaine, le développement de gated communities et de pseudo gated communities, ainsi que de nouveaux pôles d'infrastructures tertiaires de haut niveau situés en périphérie ont permis la naissance d'un archipel urbain. Ce travail est consacré à l'étude des processus ainsi que des acteurs qui en sont à l'origine. Il apparaît clairement que l'hétérogénéité des structures correspond à la diversité des acteurs. Dans le cas de Madrid toutefois, il n'y a pas à craindre de dissolution du noyau urbain.

Processes of Post-suburbanisation are manifold in Madrid. The city-region has been transformed in a breathtaking speed due to various factors: the economic restructuring processes after the end of the Franco regime, the accession to the EU and the following economic and social changes, and the replacement of former conceptions of spatial planning by more liberal and market driven ones. Although the whole city region has been transformed by now, the most significant changes can be found in the urban-rural periphery. Processes of social segregation and fragmentation, the rise of gated communities and pseudo-gated communities and the establishment of new tertiary nodes in the periphery are responsible for the formation of an urban archipelago. The paper analyses these processes and the actors who are responsible for the production of new spaces. The heterogeneity of the structures corresponds to complexity of the actors and the procedures. In the case of Madrid, despite the rise of multifaceted pos-suburban structures a disintegration of the urban core is not to be feared.

In Madrid lassen sich die Prozesse der Post-Suburbanisierung in vielen Erscheinungsformen beobachten. Die ökonomische Restrukturierung nach Ende der Franco-Diktatur, der Beitritt zur Europäischen Union und der in Folge vollzogene gesellschaftliche und ökonomische Wandel, aber auch die Ablösung früherer Raumordnungskonzeptionen durch freiere und ökonomisch determinierte Prozesse haben die Stadt in atemberaubenden Tempo verändert. Dieser Wandel umfasste die gesamte Stadtregion, ist aber in der urban-ruralen Peripherie besonders signifikant. Soziale Segregation Fragmentierung, die Entstehung von Gated Communities und Pseudo-Gated Communities und neue Knoten tertiärer Einrichtungen an der Peripherie haben ein urbanes Archipel entstehen lassen. Der Beitrag untersucht diese Prozesse auch hinsichtlich der Akteure, die sie bewirken. Es wird deutlich, dass die Heterogenität der Strukturen mit der Vielfalt der Handlungsträger korrespondiert. Die Auflösung des Stadtkerns ist im Fall von Madrid jedoch nicht zu befürchten.

\section{INDEX}

Mots-clés : Espagne, fragmentation, géographie urbaine, Madrid, postsuburbia, zone rurbaine Keywords : fragmentation, Madrid, postsuburbia, Spain, urban geography, urban-rural zone Schlüsselwörter : Fragmentierung, Madrid, Postsuburbia, Spanien, städtische Geographie, urban-rurale Zone 
AUTEUR

\section{RAINER WEHRHAHN}

Geographisches Institut, RWTH Aachen, Templergraben 55, D-52 056 Aachen 